\title{
How people present symptoms to health services: a theory-based content analysis
}

\author{
Barbara Farquharson, Marie Johnston and Carol Bugge
}

\author{
ABSTRACT

\section{Background} \\ How people present symptoms to health services may \\ influence the care they subsequently receive. \\ Leventhal's Commonsense Model of Self-Regulation \\ (CS-SRM) posits that individuals develop cognitive \\ illness representations elaborated around five main \\ components (identity, timeline, cause, consequences, \\ and cure/control), coherence, and emotional \\ representations.

\section{Aim} \\ To examine whether initial presentations to health \\ services consist of the components of illness \\ representation proposed by the CS-SRM.

\section{Design and setting} \\ A CS-SRM-based content analysis of calls to the \\ Scottish national telephone advice service, NHS 24. \\ Method \\ A random sample of callers to NHS 24 was identified. \\ A quota sample of 60 consented to participate. Their \\ consultations were transcribed verbatim. Responses to \\ an initial open question regarding the reason for calling \\ were identified and divided into 'meaning units'. A \\ coding schedule was developed using Leventhal's \\ definitions of illness representations. Meaning units \\ were coded independently by two researchers. \\ Results \\ Fifty-nine eligible initial presentations contained \\ between 1 and 13 coding units, $($ mean $=4)$. A total of \\ 230 coding units were available for coding. Overall, \\ 202 (88\%) coding units were coded to at least one \\ component of illness representation. All 59 (100\%) \\ participants made reference to identity, 26 (44\%) to \\ timeline, nine (15\%) to cause, eight (14\%) to \\ consequences, $22(37 \%)$ to cure/control, and 11 (19\%) \\ to the degree of coherence. Emotional representations \\ were identified in six (10\%) participants' presentations.

\section{Conclusion} \\ Leventhal's CS-SRM accounts for a large proportion of \\ initial presentations to health services. Most people \\ offer identity plus at least one additional component of \\ illness representation. It may be necessary for \\ clinicians to prompt remaining components to obtain a \\ comprehensive understanding of patients' \\ representations of illness.

\section{Keywords} \\ consultation; symptoms; telephone triage.
}

\section{INTRODUCTION}

How people present symptoms to health services is likely to influence the care they subsequently receive, by guiding clinical assessment and thus decisions about care. It may also influence whether the clinician and patient reach a shared understanding of the presenting problem. ${ }^{1}$ This study investigates patient presentations using Leventhal's Commonsense Self-Regulation Model (CS-SRM) as a theoretical framework. ${ }^{2}$

The CS-SRM posits that individuals actively develop representations of illness based on a general pool of knowledge of illness current in culture; social communication with individuals such as health professionals or family; and personal experience of illness. It is hypothesised that a change in somatic activity, such as a symptom, stimulates a self-regulatory process whereby individuals integrate these pre-existing ideas about illness with their current bodily experiences, and that these influence coping. The processing system consists of two parallel pathways. One involves the creation of a cognitive illness representation or 'mental picture' of a health threat and the development of a coping plan. The other pathway involves the creation of an emotional representation

B Farquharson, $B S c, M S c, P h D, R G N$, research fellow, school of psychology; M Johnston, BSc, PhD, DipClinPsych, professor emeritus, College of Life Sciences and Medicine, University of Aberdeen, Aberdeen. C Bugge, MSc, PhD, BN, AdvDipChild Dev, RN, RSCN, senior lecturer, School of Nursing, Midwifery and Health, University of Stirling, Stirling.

Address for correspondence

Dr Barbara Farquharson, University of Aberdeen, School of Psychology, William Guild Building, Aberdeen, AB24 2UB. E-mail: b.farquharson@abdn.ac.uk

Submitted: 6 October 2009; Editor's response: 15 January 2010; final acceptance: 18 June 2010.

(C)British Journal of General Practice 2011; 61: 267-273. 


\section{How this fits in}

How people present symptoms to health services is likely to influence the care they subsequently receive. The extent to which people reveal the components of illness representations within their initial presentations to health services has not previously been explored. The results of this study suggest that most people offer identity of the problem plus at least one additional component of illness representation. Clinicians may need to prompt for the remaining components to achieve a comprehensive understanding of patients' representations of illness. people's initial presentations to NHS 24. NHS 24 was the setting for the research.

\section{Participants}

A quota sample of 60 patients was recruited between May 2005 and December 2005. Thirty were patients with possible symptoms of acute coronary syndrome (as members of this group were the subject of a related study), and 30 were randomly selected from all calls to NHS 24 involving adults. The inclusion criteria were:

- a call had been handled and recorded by NHS 24;

- the patient and NHS 24 staff members had given consent for their voice recording to be used for the purposes of the study; and

- any third party involved in the call had not declined consent.

The exclusion criteria were: demonstrated that the content of illness representations is elaborated around five main components, namely: identity, timeline, cause, consequences, and cure/control. ${ }^{3-5}$ In addition, coherence, or the degree to which someone's illness representation makes sense, has been identified as important. $^{6}$

Elicitation of patients' illness representations by clinicians may lead to improved adherence with advice, ${ }^{7,8}$ improved communication, ${ }^{9}$ and increased patient satisfaction. ${ }^{10}$ However, research exploring the extent to which the components of illness representation are communicated in people's presentations to health professionals is lacking.

NHS 24 is a service within Scotland that provides health-related advice and information via the telephone. During the out-of-hours period, all calls to GPs in Scotland are coordinated by NHS 24 and automatically recorded. (The out-of-hours period varies slightly in different localities, but is usually from 6pm to 8am weekdays, and all day and night at weekends and public holidays.) As such, NHS 24 provides an ideal opportunity to study what people say at the time of seeking medical help, among a representative clinical population, without reliance on recall.

\section{Research questions}

1. Do people's initial presentations to health services consist of the components of illness representation proposed by the CS-SRM?

2. Are there further components evident within presentations unaccounted for by the model?

\section{METHOD}

Design and setting

A CS-SRM theory-based content analysis of
- any calls involving children aged 16 years or under;

- calls made by a health professional (as the aim was to explore lay perspectives of illness); and

- calls where the records indicated the patient may be suicidal (due to the potential risks in contacting such a vulnerable group).

\section{Procedures}

Ethics. To utilise the recordings of calls to NHS 24 for research, informed consent from all parties involved was required. Firstly, all staff were approached and invited to give consent for the recordings of calls they had been involved in to be used in the study. Then, all calls which both fulfilled the inclusion criteria and were handled exclusively by consenting staff members were identified, and a random selection of patients approached by letter and invited to participate.

Data collection. Where patients gave their consent, the recording of the original call was retrieved and transcribed verbatim (omitting any personally identifiable information such as name, address, date of birth). The response of participants to the call handler's initial open question (for example, 'Hello, you are through to NHS 24, can you tell me briefly why you are calling?') and to any subsequent nondirective prompts such as 'yes' or 'uh-huh' were identified for analysis. These responses were considered of particular interest, as the content was determined freely by participants and not prompted by NHS 24 staff questions. It is considered that these data are particularly valuable as they reflect how people articulate their reasons for seeking medical help in the context of a real-life health threat but 
without clinician (or researcher) contamination. ${ }^{11}$ The responses were then divided into distinct 'thoughts'. Distinct thoughts constituted either whole sentences or portions of sentences where individual thoughts were delimited by pauses, 'but' or hesitations. These constituted the coding units.

Coding. A coding scheme was developed in conjunction with a colleague doing related work, using definitions from a publication by Leventhal and colleagues, ${ }^{12}$ and a paper describing a questionnaire designed to measure illness representations. ${ }^{13}$ The definitions used are summarised in Box 1. A residual 'other' category was used to code units that did not relate to any of the predefined codes relating to CS-SRM.

Analysis. Each coding unit was coded as present or absent in relation to the components of illness representation. Fifty per cent of the transcriptions $(n=30)$ were independently coded in order to evaluate reliability. Krippendorff's $\alpha$ was used to evaluate inter-rater reliability for each construct. ${ }^{14}$ As shown in Table 1, adequate reliability (that is, $\alpha>0.7$ ) was achieved between the two raters for all constructs, with the exception of coherence, which was $<0.6$. Results reported are based on the lead author's original coding.

\section{RESULTS}

\section{Participation}

A total of 315 patients were invited to participate. Overall, $64(20 \%)$ gave consent for the recording of their call to be transcribed (the first 60 were used). This very low participation rate may have been due to a number of factors (for example, there was a narrow time window for participation, the group with possible symptoms of acute coronary syndrome may have been very unwell, and there was no face-to-face contact or ongoing relationship with NHS 24). To assess for potential bias, participants were compared with non-participants across a number of key variables. One transcription was excluded as the call handler's opening question was not an open question; thus, the total number of participants was 59.

There were no significant differences between participants and non-participants in relation to age, number of previous calls, sex, history of coronary heart disease, or whether or not the patient received an emergency response from NHS 24 (Table 2). However, those living in areas of higher deprivation and for whom the call to NHS 24 was made by someone else were significantly less likely to participate.

Nevertheless, participation from a broad range of ages (18-91 years; mean $=55$ years; standard

\section{Box 1. Definitions of CS-SRM constructs.}

Identity $^{12}$

Refers to the category, name or label, and the experience of symptoms, changes in function and visible signs. The combination of abstract and concrete experiential features 'define' or identify the disease.

\section{Timeline $^{12}$}

The duration that is expected and/or perceived with respect to the onset and duration of an illness both with and without effective treatment. Timelines are represented abstractly as clock and calendar time and concretely as experienced or felt time.

\section{Cause $^{12}$}

Reflects the perception of the single or complex set of events that are perceived as responsible for disease onset.

\section{Consequences $^{12}$}

The set of expected and perceived physical/functional, personal, and social and economic factors that are impacted by the illness.

\section{Cure/control ${ }^{12}$}

Refers to the expectation that a specific disease can be cured or controlled by the body's own defences and/or in conjunction with expert intervention, and the actual experience of the effects of these interventions on specific features (symptoms and/or test results) of disease.

\section{Coherence $^{13}$}

Whether or not people understand or have a clear picture of their illness.

\section{Emotional representation ${ }^{13}$}

An emotional representation generated by the illness.

deviation [SD] 21.05) and socioeconomic classifications was achieved (Figure 1 ). ${ }^{15}$ For $32 \%$ of participants $(n=19)$, it was their first call to NHS 24 . In $70 \%$ of cases $(n=45)$, the participant called on their own behalf. However, in the remaining $30 \%$ of cases $(n=19)$ someone else called NHS 24 on the patient's behalf.

Calls with outcomes ranging from 'self-care advice' to '999 ambulance' were represented (Figure 2).

\section{IIIness representations}

The 59 eligible initial presentations contained between 1 and 13 individual coding units, (mean $=4$ ).

Table 1. Inter-rater reliability.

\begin{tabular}{lccc} 
& Number of units coded & Krippendorff's $\alpha$ & $95 \% \mathrm{Cl}$ \\
\hline Identity & 109 & 0.865 & 0.770 to 0.962 \\
\hline Timeline & 109 & 0.865 & 0.730 to 0.973 \\
\hline Cause & 109 & 0.696 & 0.392 to 0.924 \\
\hline Consequences & 109 & 0.791 & 0.478 to 1.000 \\
\hline Cure/control & 109 & 0.840 & 0.707 to 0.946 \\
\hline Coherence & 109 & 0.593 & 0.186 to 0.919 \\
\hline Emotion & 109 & 1.000 & 0.000 to 1.000 \\
\hline Other & 109 & 0.809 & 0.555 to 1.000 \\
\hline
\end{tabular}


Table 2. Comparison of participants and non-participants.

\begin{tabular}{|c|c|c|c|c|}
\hline & Participants, $n=59$ & Non-participants, $n=256$ & & \\
\hline & Mean (SD) & Mean (SD) & Difference, 95\% Cl & $P$-value \\
\hline Age, years & $50.54(17.00)$ & $54.74(21.05)$ & -0.91 to 9.30 & ns \\
\hline \multirow[t]{2}{*}{ Number of previous calls } & $4.00(5.12)$ & $7.84(51.20)$ & -9.30 to 16.99 & ns \\
\hline & $n(\%)$ & $n(\%)$ & df & \\
\hline Male, $n$ & $22(37)$ & $102(40)$ & 1 & ns \\
\hline Documented history of CHD, $n$ & $11(18)$ & $47(19)$ & 1 & ns \\
\hline Emergency response, $n$ & $21(36)$ & $88(34)$ & 1 & ns \\
\hline \multirow[t]{2}{*}{ Caller involved, $n$} & $16(27)$ & $161(63)$ & 1 & $<0.001$ \\
\hline & & $F$ & df & \\
\hline DEPCAT score & & 2.978 & 6306 & $0.008^{a}$ \\
\hline
\end{tabular}

$C H D=$ coronary heart disease. DEPCAT = deprivation category. $d f=$ degrees of freedom. $n s=$ not significant.

$S D=$ standard deviation .

A total of 230 coding units were available for coding. Overall, 202 (88\%) coding units were coded to at least one component of illness representation (Table 3).

Participant identification numbers are in brackets following illustrative quotations.

Identity. All 59 participants referred to identity; most referring to their experience of symptoms, for example: 'I've been getting pains across my chest' (4009), but many $(n=22)$ also used labels (for

Figure 1. DEPCAT classification of participants: higher DEPCAT scores represent postcode sectors with higher deprivation.

Figure 2. Outcome of participants' calls.
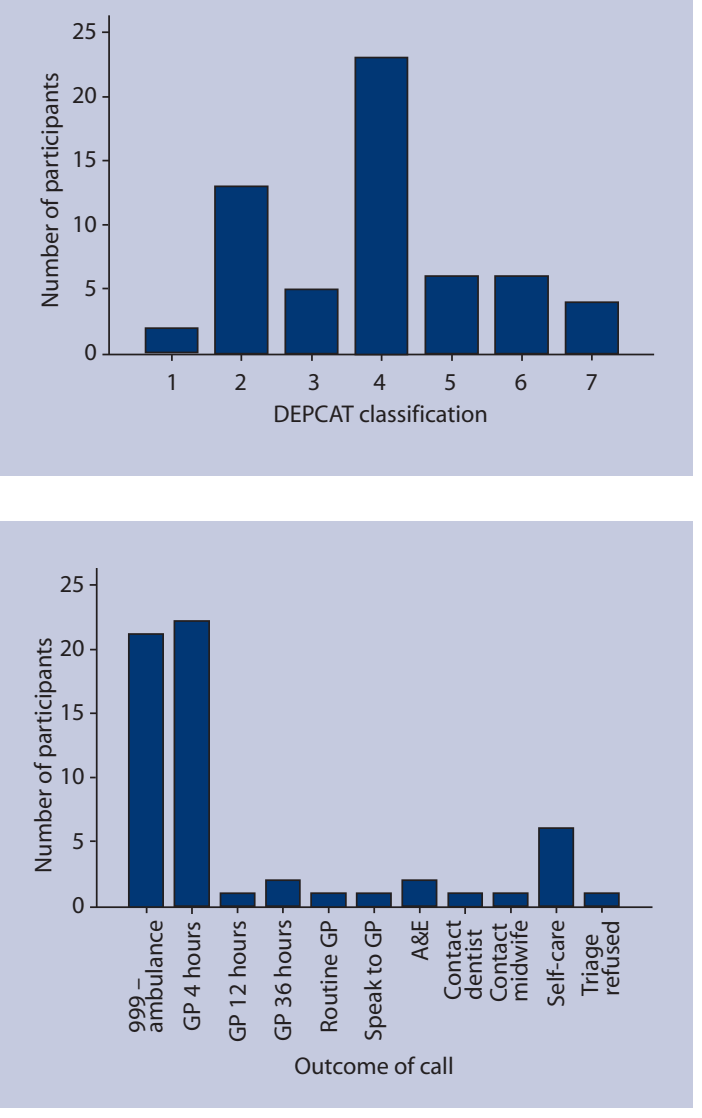

example, 'cystitis' ([4076]), and 15 referred to visible signs: 'she is very pale' (5014).

Timeline. Most of the 26 participants who referred to timeline, referred to the duration of symptoms; for example: 'He's been up the whole night' (4040). Some indicated precise times of the onset of symptoms: 'It started actually at 8 o'clock this morning; I had shooting pains down my left hand' (4045). One participant volunteered that their symptoms were cyclical in nature: '... and it seems to come maybe every 4 or 5 weeks' (5017).

Cause. Few participants $(n=9)$ mentioned cause in their responses as to why they were calling. Of those who did, a few clearly articulated possible causes for the symptoms: 'l'm calling because em my husband is experiencing symptoms which almost certainly are from his heart' (5010).

In some cases it appeared that pre-existing knowledge or experience led participants to suspect a particular cause: 'See as soon as I hear about chest pains I think of course about the heart and everything like that, you know' (5014).

Consequences. Again, few $(n=8)$ participants referred to the likely consequences of their symptoms. Where consequences were mentioned, they tended to relate to physical functioning "I was in bed and I couldn't get up to open the doors and that' (4015) and 'She just collapsed on the stairs and can't move' (4077).

References to personal and social consequences were also identified, but instances relating to economic consequences were not: 'Um I've injured my left hand and um unfortunately I am flying out at 3 o'clock in the morning ...' (4006).

Cure/control. Twenty-two participants made 
reference to cure/control. Some related to the request for medical assistance, some stating what they thought was required: ' $\mathrm{Em}$ I was wondering if I could em be seen by a doctor. So I really need to see if ... I think what I might need is a hand-brace?' (4006).

Others sought advice as to what would be an appropriate course of action: 'I just wanted to see if I should go to the hospital or not as I have just been feeling a wee bit sick' (4020).

A number mentioned attempts they had already made to control the symptoms: 'Yeah, em l've had a constant headache for 2 days and paracetamol is, paracetamol is just not shifting it and my usual migraine tablets that I take aren't shifting it either' (5063).

Coherence. A number of participants made statements that suggested that they had a clear understanding of their symptoms: 'I'm calling because em my husband is experiencing symptoms which almost certainly are from his heart' (5010).

However, other participants' statements suggested less coherence in relation to their symptoms, as in the example of the following three statements from a single participant:

'But she says she wakened up with chest pains at 6 o'clock but we don't know if she has maybe pulled a muscle or if its flu I don't know ...'

'... maybe it is nothing but she wakened up with a pain down her left arm at 4 o'clock this morning ...'

'... she says her gums have been bleeding but I don't know if that's anything to do with it.' (5014)

Emotion. Emotional responses to symptoms were evident in a small number of participants' $(n=6)$ responses to the initial open questions. These ranged from mild concern to high distress:

'Now what it is, I'm just a wee bit worried.' (5014)

'I'm extremely worried about him.' (4075)

'Em, well he has been in the hospital with em ... eh oh dear, oh God ... angina attack.' (5024)

Other. There were $28(12 \%)$ coding units that could not be allocated to any of the predefined codes. Inductive analysis of the units that could not be allocated to the CS-SRM codes revealed that most ( $n=16)$ consisted of a statement in which participants introduced either themselves or the
Table 3. Number of meaning units and individuals referring to components of illness representation.

\begin{tabular}{|c|c|c|c|c|}
\hline & \multicolumn{2}{|c|}{$\begin{array}{l}\text { Units coded, } \\
\qquad n=230\end{array}$} & \multicolumn{2}{|c|}{$\begin{array}{c}\text { Indivduals, } \\
n=59\end{array}$} \\
\hline & $n$ & $\%$ & $n$ & $\%$ \\
\hline Identity & 131 & 56 & 59 & 100 \\
\hline Timeline & 48 & 21 & 26 & 44 \\
\hline Cause & 10 & 4 & 9 & 15 \\
\hline Consequences & 9 & 4 & 8 & 14 \\
\hline Cure/control & 46 & 20 & 22 & 37 \\
\hline Coherence & 13 & 6 & 11 & 19 \\
\hline Emotion & 8 & 3 & 6 & 10 \\
\hline
\end{tabular}

person they were calling about; for example: 'Good evening, my name is ...' (4060) and 'Hi there. It's em ... it's actually my grandfather' (1050). A further seven coding units were composed of odd words or phrases, usually hesitations within the dialogue; for example: 'And it was em ...' (4020) and 'I'll tell you what it is' (5033).

However, a further four coding units appeared to relate to the context in which the symptoms were occurring:

I've just come in and my Mum has just phoned me.' (4055)

'She is just sitting beside me just now.' (4068)

'She lives on her own, right.' (5014)

\section{Prevalence of illness representation components}

The number of participants making reference to the respective components of illness representation is summarised in Table 3.

Participants volunteered between one and six components of illness representation (mean $=2.24$, $\mathrm{SD}=1.18$ )

The results of this study are compared with those of previous work in this area, in Table 4. Lau et al

Table 4. Prevalence (\%) of components of illness representation. Comparison with Lau et al (1989). ${ }^{4}$

\begin{tabular}{lcc} 
& Lau et al (1989) & Current study \\
\hline Identity & 96 & 100 \\
\hline Timeline & 49 & 44 \\
\hline Cause & 28 & 15 \\
\hline Consequences & 33 & 14 \\
\hline Cure control & 32 & 37 \\
\hline Coherence & Not investigated & 19 \\
\hline Emotion & Not investigated & 10 \\
\hline
\end{tabular}


asked participants, on a number of occasions, to describe everything they remembered about a recent illness. ${ }^{4}$ The results they obtained on first administration are presented for comparison.

It can be seen that similar proportions of participants referred to identity and timeline. However, a much smaller percentage of participants in the current study made reference to both consequences and cause than in the study by Lau and colleagues. ${ }^{4}$ Results regarding cure/control were similar in the two studies, while coherence and emotion were not reported by Lau and colleagues. ${ }^{4}$

\section{DISCUSSION}

\section{Summary of main findings}

A large proportion (88\%) of the coding units were related to components of illness representation. If coding units where the participant simply introduced themselves (or the patient) were excluded, the proportion was even higher (95\%). Thus, the CSSRM successfully accounted for the content of participants' initial symptom presentations.

The majority of the remaining $5 \%$ coding units that could not be coded to a component of illness representation tended to relate to the context in which the symptoms occurred. Thus, it may be that people include context as a component when articulating their representation of illness to health services. However, it is also possible that people rehearse the context in order to aid their recall of the events surrounding the onset of symptoms, ${ }^{16}$ that is, that context is not part of the illness representation but rather a cue to recalling illness representations.

\section{Strengths and limitations of the study}

The major strength of the methodology was that participants' transcription data were provided at the actual time of seeking help and could not have been influenced by subsequent diagnosis. Thus it is considered that these data accurately reflect how people articulate their reasons for seeking medical help in the context of a real-life health threat but, importantly, without clinician or researcher contamination.

The main limitation of the study was the low participation rate (20\%), which is likely to have resulted in some bias in the sample. Furthermore, reasons for non-participation were not systematically collected, which limits the ability to assess further the likely impact of the low participation rate. However, a variety of information about potential participants was available at NHS 24, which allowed assessments of non-responder bias to be undertaken and accounted for in interpretation of results.

During analysis, no differentiation was made between calls made by the patient and those made by a third party, which could be considered a limitation of the approach taken.

Coding was undertaken by the investigator and thus it was possible that the results could be subject to bias in relation to the investigator's interpretation. However, the data were independently coded by a second investigator, and coding was found to be highly reliable between the two coders, even after correcting for chance agreement. Furthermore, the use of N-Vivo software ensured that the coding of data is readily available for further scrutiny and independent replication of analysis.

\section{Comparison with existing literature}

The difference in prevalence of consequence and cause statements in contrast to results obtained by Lau et $a l^{4}$ may be related to the difference in context and methodology. Participants recalling a previous, resolved illness may be more likely to consider aspects such as consequences and cause than those experiencing current symptoms. Alternatively, it may be that people are particularly reluctant to reveal their ideas about cause or consequence to health services. However, it must also be borne in mind that only participants' responses to the initial open question constituted the subject of this analysis. While these data are particularly valuable as they are largely uncontaminated by either the researcher's or professionals' questions, they represent a small proportion of the overall clinical presentation. It is possible that participants may be more likely to discuss their ideas about cause and consequences later in consultations. Analysis of the remainder of the transcripts is required (and indeed planned), in order to establish if this is the case.

\section{Implications for clinical practice and future research}

These results suggest that people spontaneously give a description of the symptoms or label they attribute to their (or someone else's) illness, and most people offer at least one additional component of illness representation. However, it may be necessary for clinicians to prompt the remaining components to obtain a fuller understanding of people's representations of their illness. A better understanding of individuals' illness representations has the potential to improve adherence with advice, ${ }^{7,8}$ improve communication, ${ }^{9}$ and lead to increased patient satisfaction. ${ }^{10}$

Leventhal's CS-SRM accounts for a large proportion of people's initial presentations to health services. To obtain a comprehensive view of 
people's representations of their illness, it may be necessary to prompt some components identified by the CS-SRM. Further research exploring the role of clinicians' questioning in eliciting illness representations during initial presentation is required.

\section{Funding body}

This research was funded by the Nursing, Midwifery and Allied Health Professionals (NMAHP) Research Training Scheme (Scotland).

\section{Ethics committee}

Fife and Forth Valley NHS Research Ethics Committee (04/S0501/74). NHS management approval was provided by NHS 24

\section{Competing interests}

The authors have stated that there are none.

\section{Acknowledgements}

Grateful thanks to the patients and staff of NHS 24 who generously gave permission for their recordings to be used in this research, and to Liz Glidewell, University of Aberdeen for her assistance with coding.

\section{Discuss this article}

Contribute and read comments about this article on the Discussion Forum: http://www.rcgp.org.uk/bjgp-discuss

\section{REFERENCES}

1. Bugge C, Entwistle VA, Watt IS. The significance for decision-making of information that is not exchanged by patients and health professionals during consultations. Soc Sci Med 2006; 63(8): 2065-2078.

2. Leventhal $H$, Meyer D, Nerenz D. The common sense representation of illness danger. In: Rachman S (ed). Contributions to medical psychology. 1st edn, Vol 2. Oxford: Pergamon Press, 1980: 7-30.

3. Lau RR, Hartman KA. Common sense representations of common illnesses. Health Psychol 1983; 2(2): 167-185.
4. Lau RR, Bernard TM, Hartman K. Further explorations of commonsense representations of common illnesses. Health Psychol 1989; 8(2): 195-219.

5. Bishop GD, Briede C, Cavazos L, et al. Processing of illness information: the role of disease prototypes. Basic Appl Soc Psych 1987; 8: $21-43$.

6. Hall S, Weinman J, Marteau T. The motivating impact of informing women smokers of a link between smoking and cervical cancer: the role of coherence. Health Psychol 2004; 23(4): 419-424.

7. Horne R, Weinman J. Self-regulation and self-management in asthma: exploring the role of illness perceptions and treatment beliefs in explaining non-adherence to preventer medication. Psychol Health 2002; 17(1): 17-32.

8. Cooper AF, Weinman J, Hankins M, et al. Assessing patients' beliefs about cardiac rehabilitation as a basis for predicting attendence after acute myocardial infarction. BMJ 2007; 93(1): 53-58.

9. De Ridder DTD, Theuissen NCM, van Dulmen SM. Does training general practioners to elicit patients' illness representations and action plans influence their communication as a whole? Patient Educ Couns 2007; 66(3): 327-336.

10. Lang F, Floyd M, Beine K, Buck P. Sequenced questioning to elicit the patient's perspective on illness: effects on information disclosure, patient satisfaction and time expenditure. Fam Med 2002; 34(5): 325-330.

11. Webb EJ, Campbell DT, Schwartz RD, Sechrest L. Unobtrusive measures. Newbury Park, CA: Sage Publications Inc, 1999.

12. Leventhal H, Forster RS, Leventhal E. Self-regulation of health threats, affect and the self: lessons from the elderly. In: Aldwin C, Park CL, Spiro AI (eds). Handbook of health psychology and aging. New York, NY: Guilford Publications, 2007, pp: 341-366.

13. Moss-Morris R, Weinman J, Petrie K, et al. The revised illnes perception questionnaire (IPQ-R). Psychol Health 2002; 17(1): 1-16.

14. Hayes AF, Krippendorff $K$. Answering the call for a standard reliability measure for coding data. Commun Methods Meas 2007; 1(1): 77-89.

15. McLoone P. Carstairs scores for Scottish postcode sectors from the 2001 Census. Glasgow: MRC Social and Public Health Sciences Unit, March 2004. http://www.sphsu.mrc.ac.uk/files/File/library/ other\%20reports/Carstairs_report.pdf (accessed 11 Mar 2011).

16. Smith S. Theoretical principles of context-dependent memory. In Morris P, Gruneberg MM (eds). Theoretical aspects of memory. 2nd edn. London: Routledge, 1994, pp: 168-195. 